\title{
On the Gravitomagnetic Time Delay
}

\author{
I. Ciufolini ${ }^{\text {a }}$, S. Kopeikin ${ }^{\text {b }}$, B. Mashhoon ${ }^{\text {b,* }}$, F. Ricci $^{\text {c }}$ \\ ${ }^{a}$ Dipartimento di Ingegneria dell'Innovazione, Università di Lecce, Via Monteroni, \\ 73100 Lecce, Italy \\ ${ }^{\mathrm{b}}$ Department of Physics and Astronomy, University of Missouri-Columbia, \\ Columbia, Missouri 65211, USA \\ 'Dipartimento di Fisica, Università di Roma, 'La Sapienza', Piazzale Aldo Moro \\ 5, 00185 Roma, Italy
}

\begin{abstract}
We study the gravitational time delay in ray propagation due to rotating masses in the linear approximation of general relativity. Simple expressions are given for the gravitomagnetic time delay that occurs when rays of radiation cross a slowly rotating shell, equation (14), and propagate in the field of a distant rotating source, equation (16). Moreover, we calculate the local gravitational time delay in the Gödel universe. The observational consequences of these results in the case of weak gravitational lensing are discussed.
\end{abstract}

Key words: gravitomagnetism, time delay, gravitational lensing PACS: 04.20.Cv; 95.30.S

\section{Introduction}

Consider the gravitational field of astronomical sources in the linear approximation of general relativity. The spacetime metric may be expressed in the quasi-inertial coordinates $x^{\mu}=(c t, \mathbf{x})$ as $g_{\mu \nu}=\eta_{\mu \nu}+h_{\mu \nu}$, where $\eta_{\mu \nu}$ is the Minkowski metric with signature +2 . In the absence of perturbing potentials $h_{\mu \nu}(x)$, rays of electromagnetic radiation propagate along straight lines defined by $d x^{i} / d t=c \hat{k}^{i}$, where $\hat{\mathbf{k}}$ is the constant unit propagation vector of the signal. These zeroth-order null geodesics will be employed throughout this work. In the exterior gravitational field of astronomical sources, however, the rays

* Corresponding author

Email address: mashhoonb@missouri.edu (B. Mashhoon). 
bend due to the attraction of gravity as they follow geodesics of the spacetime manifold that are null. It turns out that in order to evaluate the gravitational time delay to first order in $h_{\mu \nu}$ only the null condition is required, i.e.

$$
g_{\mu \nu} d x^{\mu} d x^{\nu}=0
$$

We are interested in the consequences of this condition for the propagation of a ray on a background global inertial frame. Therefore, it follows from equation (1) that $c^{2} d t^{2}-|d \mathbf{x}|^{2}=h_{\mu \nu} d x^{\mu} d x^{\nu}$, where $|d \mathbf{x}|^{2}=\delta_{i j} d x^{i} d x^{j}$. To first order in the perturbation, the bending of the ray may be neglected in evaluating the right-hand side of this result, which may be written as $c^{2} h_{\alpha \beta} k^{\alpha} k^{\beta} d t^{2}$. Here $k^{\alpha}=(1, \hat{\mathbf{k}})$ is such that $\eta_{\mu \nu} k^{\mu} k^{\nu}=0$. One may therefore write equation (1) in the form

$$
c d t=\left(1+\frac{1}{2} h_{\alpha \beta} k^{\alpha} k^{\beta}\right)|d \mathbf{x}| .
$$

Let the ray propagate from a point $P_{1}:\left(c t_{1}, \mathbf{x}_{1}\right)$ to a point $P_{2}:\left(c t_{2}, \mathbf{x}_{2}\right)$ in the background inertial frame; then,

$$
t_{2}-t_{1}=\frac{1}{c}\left|\mathbf{x}_{2}-\mathbf{x}_{1}\right|+\frac{1}{2 c} k^{\alpha} k^{\beta} \int_{P_{1}}^{P_{2}} h_{\alpha \beta}(x) d l
$$

where $d l=|d \mathbf{x}|$ denotes the Euclidean length element along the straight line that joins $P_{1}$ to $P_{2}$. It follows from equation (3) that the gravitational time delay $\Delta$ is given by

$$
\Delta_{G}=\frac{1}{2 c} \int_{P_{1}}^{P_{2}} h_{\alpha \beta}(x) k^{\alpha} k^{\beta} d l .
$$

Note that in this expression $h_{\alpha \beta}$ may be replaced by $\bar{h}_{\alpha \beta}=h_{\alpha \beta}-\frac{1}{2} \eta_{\alpha \beta} h$, since $\eta_{\alpha \beta} k^{\alpha} k^{\beta}=0$. Here $h=\operatorname{tr}\left(h_{\mu \nu}\right)$. Moreover, under a gauge transformation of the gravitational potential $h_{\mu \nu} \rightarrow h_{\mu \nu}+\epsilon_{\mu, \nu}+\epsilon_{\nu, \mu}$, corresponding to an infinitesimal coordinate transformation $x^{\mu} \rightarrow x^{\mu}-\epsilon^{\mu}$, equation (4) simply gives the time delay in the new coordinates.

In physical circumstances where the linearized Einstein equations are applicable, the time delay $\Delta_{G}$ may be combined with the proper time $\tau$ measured by an observer moving with velocity $\mathbf{v}=c \boldsymbol{\beta}$,

$$
d \tau=c d t \sqrt{-g_{00}-2 g_{o i} \beta^{i}-g_{i j} \beta^{i} \beta^{j}}
$$


in order to arrive at physically measurable predictions of the theory.

The gravitational time delay was studied by Shapiro [1] in terms of radar echo delay and subsequently by a number of investigators (see [2]-[8] and references cited therein). The general derivation of the linear effect (4) given here is free of special assumptions and can be employed in most situations of physical interest.

The gravitomagnetic time delay is discussed in sections 2 and 3. The Gödel universe is considered in section 4; we study the local gravitational time delay in this rotating universe. In section 5 we analyze the gravitomagnetic time delay in different images of the same source due to gravitational lensing. Finally, section 6 contains a brief discussion of our results.

\section{Gravitoelectromagnetism}

Let us now consider the exterior field of slowly moving sources such that the trace-reversed potential $\bar{h}_{\mu \nu}$ satisfies $\square \bar{h}_{\mu \nu}=-\left(16 \pi G / c^{4}\right) T_{\mu \nu}$, once the gauge condition $\bar{h}_{, \nu}^{\mu \nu}=0$ has been imposed. We are interested in the particular retarded solution

$$
\bar{h}_{\mu \nu}=\frac{4 G}{c^{4}} \int \frac{T_{\mu \nu}\left(c t-\left|\mathbf{x}-\mathbf{x}^{\prime}\right|, \mathbf{x}^{\prime}\right)}{\left|\mathbf{x}-\mathbf{x}^{\prime}\right|} d^{3} \mathbf{x}^{\prime},
$$

which can be used directly in equation (4) to compute the gravitational time delay. The generalization of the Shapiro effect to time-dependent situations has been the subject of a recent investigation [6].

We confine our study here to the stationary field of a slowly rotating astronomical body such that $\bar{h}_{00}=4 \Phi_{g} / c^{2}, \bar{h}_{0 i}=-2\left(\mathbf{A}_{g}\right)_{i} / c^{2}$ and $\bar{h}_{i j}=O\left(c^{-4}\right)$, where $\Phi_{g}(x)$ is the gravitoelectric potential, $\mathbf{A}_{g}(x)$ is the gravitomagnetic vector potential $\left(\boldsymbol{\nabla} \cdot \mathbf{A}_{g}=0\right)$ and we neglect all terms of order $c^{-4}$ including the spatial potentials $\bar{h}_{i j}$. Far from the source

$$
\Phi_{g} \sim \frac{G M}{r}, \quad \mathbf{A}_{g} \sim \frac{G}{c} \frac{\mathbf{J} \times \mathbf{r}}{r^{3}}
$$

where $M$ and $J$ are the total mass and angular momentum of the source. We define the gravitoelectric field to be $\mathbf{E}_{g}=-\nabla \Phi_{g}$ and the gravitomagnetic field to be $\mathbf{B}_{g}=\boldsymbol{\nabla} \times \mathbf{A}_{g}$. In developing this analogy with electrodynamics, one encounters extra numerical factors that cannot be avoided. For instance, the field equation for the standard gravitomagnetic vector potential has an extra factor of -4 in comparison with Maxwell's theory [2]. In this connection, we 
use the following convention that is consistent with the gravitational Larmor theorem [9]: The standard formulas of classical electrodynamics are applicable, except that for a source of inertial mass $M$, the gravitoelectric charge is $M$ and the gravitomagnetic charge is $2 M$ (in units such that $G=1$ ). On the other hand, for a test particle of mass $m$, the gravitoelectric charge is $-m$ and the gravitomagnetic charge is $-2 m$. The source and the test particle have opposite charges to ensure that gravity is attractive. Moreover, the ratio of the gravitomagnetic charge to the gravitoelectric charge is always 2, since linearized gravity is a spin-2 field.

We find from equation (4) that $\Delta_{G}=\Delta_{G E}+\Delta_{G M}$, where

$$
\Delta_{G E}=\frac{2}{c^{3}} \int_{P_{1}}^{P_{2}} \Phi_{g} d l
$$

is the Shapiro time delay and

$$
\Delta_{G M}=-\frac{2}{c^{3}} \int_{P_{1}}^{P_{2}} \mathbf{A}_{g} \cdot d \mathbf{x}
$$

is the gravitomagnetic time delay, which is simply proportional to the line integral of the gravitomagnetic vector potential.

Let us now suppose that we can arrange via "mirrors" - these could be transponders on spacecraft - to have the rays travel on a closed trajectory as in Figure 1. Then the net gravitomagnetic time delay for a closed loop in the positive sense (i.e. counterclockwise) is

$$
\Delta_{G M}^{+}=-\frac{2}{c^{3}} \oint \mathbf{A}_{g} \cdot d \mathbf{x}=-\frac{2}{c^{3}} \int \mathbf{B}_{\mathbf{g}} \cdot d \mathbf{S}
$$

which is simply proportional to the gravitomagnetic flux threaded by a surface $S$ whose boundary is the closed loop under consideration $\square$. Imagine now a point $P$ on the trajectory (cf. Figure 1). If the rays travel along the same path but in the opposite direction, the net gravitoelectric time delay measured at $P$ will be the same while the gravitomagnetic time delay will change sign, $\Delta_{G M}^{-}=-\Delta_{G M}^{+}$. Thus the total time difference at $P$ for the rays to go around the loop in opposite directions is given by

$$
t_{+}-t_{-}=-\frac{4}{c^{3}} \oint \mathbf{A}_{g} \cdot d \mathbf{x}=-\frac{4}{c^{3}} \int \mathbf{B}_{\mathbf{g}} \cdot d \mathbf{S} .
$$

$\overline{1}$ It is interesting to note that the direct echo delay (i.e. when the loop degenerates to a line) vanishes in the gravitomagnetic case as the effective area is zero. 
A different derivation of this result based on the propagation of electromagnetic radiation in the gravitational field of a rotating mass is contained in ref.

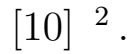

Let us now estimate this time difference for the case of GPS signals traveling around the Earth. We find that $t_{+}-t_{-} \approx-8 \pi G J_{\oplus} /\left(c^{4} R_{\oplus}\right) \approx-10^{-16} \mathrm{sec}$. By contrast, the Shapiro time delay amounts to $2 \times 10^{-10}$ sec for clocks in GPS orbit [11], [12], so that this gravitoelectric effect may be measurable in the near future. In such an experiment, the time of flight of the signal is monitored and eventually measured by a control station on the Earth [11], [12]. The corresponding gravitomagnetic effect is about two million times smaller and can be completely neglected at present. Other very small angular momentum effects in laboratory-based optical interferometry experiments have been studied in [13], [14].

\section{Gravitomagnetic time delay}

In this section, we provide explicit formulas for $\Delta_{G M}$ for the case of radiation crossing a slowly rotating thin shell of matter as well as propagating in the exterior field of a distant rotating mass.

In electrodynamics, the magnetic vector potential inside a spherical shell of uniform charge density with total charge $Q$ and radius $R_{0}$ rotating with constant frequency $\omega$ is given by $\mathbf{A}=\frac{1}{2} \mathbf{B} \times \mathbf{r}$, where $\mathbf{B}=\frac{2}{3} Q \boldsymbol{\omega} /\left(c R_{0}\right)$ is the uniform magnetic field inside the shell. The corresponding electric field vanishes. Therefore, the gravitomagnetic vector potential inside a uniform shell of mass $M$, radius $R_{0}$ and rotational frequency $\boldsymbol{\omega}$ is [15], [16]

$$
\mathbf{A}_{g}=\frac{2 G M}{3 c R_{0}} \boldsymbol{\omega} \times \mathbf{r}
$$

where the center of the shell is the spatial origin of a background inertial frame. Thus inside the shell $\mathbf{B}_{g}=4 G M \boldsymbol{\omega} /\left(3 c R_{0}\right)$ and $\mathbf{E}_{g}=0$. The gravitomagnetic time delay is therefore

$$
\Delta_{G M}=-\frac{4 G M}{3 c^{4} R_{0}} \boldsymbol{\omega} \cdot \int_{P_{1}}^{P_{2}} \mathbf{r} \times d \mathbf{r} .
$$

$\overline{2}$ A sign error in this reference must be corrected: In ref. [10], the relation $\Phi_{+}-\Phi_{-}=$ $\omega\left(t_{+}-t_{-}\right)$contains an errant minus sign. Therefore in equations (20), (21) and related discussion in section 3 of [10], the sign of $t_{+}-t_{-}$has to be reversed. 
Let $P_{1}$ and $P_{2}$ be the two points on the spherical shell indicating the points at which the ray enters and leaves the sphere, respectively. Then a simple calculation shows that

$$
\Delta_{G M}=-\frac{4 G M R_{0}}{3 c^{4}} \boldsymbol{\omega} \cdot\left(\hat{\mathbf{r}}_{1} \times \hat{\mathbf{r}}_{2}\right)
$$

where $\hat{\mathbf{r}}_{1}$ and $\hat{\mathbf{r}}_{2}$ are unit vectors indicating the positions of $P_{1}$ and $P_{2}$, respectively, from the center of the sphere. Note that the result vanishes if $\boldsymbol{\omega}, \mathbf{r}_{1}$ and $\mathbf{r}_{2}$ are in the same plane; in particular, $\Delta_{G M}=0$ if the ray passes through the center of the spherical shell.

Let us next consider the exterior field of a rotating mass with its center of mass at the origin of spatial coordinates such that $r_{1}$ and $r_{2}$ are both much larger than $2 G M / c^{2}$; then, neglecting higher-order multipole moments we find that 3

$$
\Delta_{G M}=-\frac{2 G}{c^{4}} \int_{P_{1}}^{P_{2}} \frac{\mathbf{J} \cdot(\mathbf{r} \times d \mathbf{r})}{r^{3}} .
$$

A detailed, but straightforward, calculation shows that

$$
\Delta_{G M}=-\frac{2 G J}{c^{4}}\left(\frac{1}{r_{1}}+\frac{1}{r_{2}}\right) \frac{\hat{\mathbf{J}} \cdot\left(\hat{\mathbf{r}}_{1} \times \hat{\mathbf{r}}_{2}\right)}{1+\hat{\mathbf{r}}_{1} \cdot \hat{\mathbf{r}}_{2}} .
$$

Thus $\Delta_{G M}$ also vanishes in this case if $\mathbf{J}, \mathbf{r}_{1}$ and $\mathbf{r}_{2}$ are in the same plane.

Let us note that equation (16) is exact and holds in general for the exterior of any spherically symmetric slowly rotating mass with constant J. Moreover, let $d$ be the impact parameter of the light ray; then, when $r_{1} / d>>1$ and $r_{2} / d>>1$ it is possible to show that $\Delta_{G M} \simeq-4 G \mathbf{J} \cdot \hat{\mathbf{n}} /\left(c^{4} d\right)$, where $\hat{\mathbf{n}}$ is a unit vector normal to the plane formed by $\mathbf{r}_{1}$ and $\mathbf{r}_{2}$ and directed along $\mathbf{r}_{1} \times \mathbf{r}_{2}$. Extending this calculation to the case in which the source is a thin rotating spherical shell of constant radius $R_{0}$ and the light ray penetrates the shell such that the portion of the ray inside the shell subtends an angle $\varphi$ at the center of the shell, we find from equations (14) and (16) that for $r_{1}>>R_{0}$ and $r_{2}>>R_{0}, \Delta_{G M} \simeq-4 G \mathbf{J} \cdot \hat{\mathbf{n}} f(\varphi) /\left(c^{4} d\right)$, where $f(\varphi)=\tan \left(\frac{\pi-\varphi}{4}\right)+\frac{1}{2} \sin \varphi$ varies from $f(0)=1$ to $f(\pi)=0$ as $\varphi: 0 \rightarrow \pi$. The impact parameter of the ray is given by $d=R_{0} \cos \frac{\varphi}{2}$, so that for $\varphi=0$ we recover the previous expression for $\Delta_{G M}$.

3 The general expression for the gravitomagnetic time delay in the gravitational field of higher-spin multipole moments of a stationary rotating body was derived in [4], equation (43). A sign error in formula (43) must be corrected: the overall sign of this expression must be reversed. 
In two recent papers [17], [18], the gravitomagnetic time delay in different images due to gravitational lensing has been studied and a derivation of the time delay due to the spin of an external shell has been presented. These results, together with a discussion of the separability of time delays of different origin in the images, demonstrate that the gravitomagnetic time delay would in principle be measurable in the near future. In particular, the gravitomagnetic time delay must be taken into account in the analysis of gravitational lensing time delay of some extragalactic sources, since the estimated $\Delta_{G M}$ may exceed the present measurement uncertainty of $\sim 0.5$ day in the lensing time delay; we discuss this issue in section 5 . Further measurement aspects of $\Delta_{G M}$ are explored in [19], [20]. It is hoped that the measurement of $\Delta_{G M}$ might provide information about the existence and distribution of rotating dark matter.

It would be interesting to estimate how much of the gravitational time delay between any two local points $P_{1}$ and $P_{2}$ could be due to the cosmological content of the universe. To this end, we consider the development of a Fermi coordinate system in the neighborhood of a fundamental geodesic observer in the standard FLRW model [21]. At an epoch with Hubble "constant" $H$, the gravitational time delay over any local distance $r$ compared to $r / c$ is of the order $H^{2} r^{2} / c^{2}$, which is negligibly small. A similar estimate holds for a rotating universe model as explained in the next section.

\section{Gödel universe}

The metric of the stationary and spatially homogeneous Gödel universe can be expressed as [22]

$$
d s^{2}=-d t^{2}-2 \sqrt{2} U(x) d t d y+d x^{2}-U^{2}(x) d y^{2}+d z^{2}
$$

where $U(x)=\exp (\sqrt{2} \Omega x)$ and we use units such that $c=1$ unless specified otherwise. It turns out that for this metric the Ricci curvature is given by

$R_{\mu \nu}=2 \Omega^{2} u_{\mu} u_{\nu}$, where $u^{\mu}=\delta^{\mu}{ }_{0}$ is the velocity vector of a particle at rest in space and coincides with the timelike Killing vector $\partial_{t}$. The physical content of this model may be thought of as a perfect fluid with velocity $u^{\mu}$ and constant density and pressure given by $\rho=p=\Omega^{2} /(8 \pi G)$, where $\Omega \partial_{z}$ is the vorticity vector associated with the geodesic worldlines of the fluid. Alternatively, the universe could be filled with dust of constant density $\Omega^{2} /(4 \pi G)$ together with a cosmological constant $\Lambda=-\Omega^{2}[23]$.

To study the influence of this rotating cosmos on the local physics, we establish a Fermi normal coordinate system in the neighborhood of a standard observer in this model. Imagine therefore an observer at rest in space and comoving 
with the fluid; then, the observer follows a geodesic and its velocity vector $u^{\mu}=\lambda^{\mu}{ }_{(0)}$ is the temporal axis of an orthonormal tetrad frame $\lambda^{\mu}{ }_{(\alpha)}$ that is parallel transported along the geodesic. In $(t, x, y, z)$ coordinates, such a tetrad is given by

$$
\begin{aligned}
& \lambda^{\mu}{ }_{(0)}=(1,0,0,0), \\
& \lambda^{\mu}{ }_{(1)}=\tilde{\lambda}^{\mu}{ }_{(1)} \cos \Omega t+\tilde{\lambda}_{(2)}^{\mu} \sin \Omega t, \\
& \lambda^{\mu}{ }_{(2)}=-\tilde{\lambda}^{\mu}{ }_{(1)} \sin \Omega t+\tilde{\lambda}_{(2)}^{\mu} \cos \Omega t, \\
& \lambda^{\mu}{ }_{(3)}=(0,0,0,1),
\end{aligned}
$$

where $\tilde{\lambda}_{(1)}^{\mu}$ and $\tilde{\lambda}_{(2)}^{\mu}$ are defined by

$$
\begin{aligned}
& \tilde{\lambda}^{\mu}{ }_{(1)}=(0,1,0,0), \\
& \tilde{\lambda}_{(2)}^{\mu}=\left(-\sqrt{2}, 0, U^{-1}(x), 0\right) .
\end{aligned}
$$

Here the rotation of the orthonormal triad $\lambda_{(i)}^{\mu}, i=1,2,3$, representing ideal gyroscope directions characterizing the spatial Fermi frame, about the $z$-axis with frequency $\Omega$ has been made explicit. The Fermi coordinate system assigns coordinates $X^{\mu}=(T, X, Y, Z)$ to an event $P$ in the neighborhood of the geodesic worldline under consideration as follows : there exists a unique spacelike geodesic connecting $P$ to the worldline at $P_{0}$ such that the two paths are orthogonal at $P_{0}$, i.e. $\lambda^{\mu}{ }_{(0)} \eta_{\mu}=0$, where $\eta_{\mu}$ is the unit tangent vector to the spacelike geodesic at $P_{0}$. Let $\tau$ be the proper time of the observer at $P_{0}, \sigma$ be the proper length of the spacelike geodesic segment $P_{0} P$ and $C_{i}=\eta_{\mu} \lambda_{(i)}^{\mu}$ be the direction cosines of this segment with respect to the observer's triad at $P_{0}$. Then $T=\tau, X=\sigma C_{1}, Y=\sigma C_{2}$ and $Z=\sigma C_{3}$. In these Fermi coordinates, the spacetime in the neighborhood of the observer is Minkowskian except for the cosmic tidal influence expressed by the gravitational potential ${ }^{F} h_{\mu \nu}$, i.e. $g_{\mu \nu}=\eta_{\mu \nu}+{ }^{F} h_{\mu \nu}$, where

$$
\begin{aligned}
& { }^{F} h_{00}=-{ }^{F} R_{0 i 0 j} X^{i} X^{j}, \\
& { }^{F} h_{0 i}=-\frac{2}{3}{ }^{F} R_{0 j i k} X^{j} X^{k}, \\
& { }^{F} h_{i j}=-\frac{1}{3}{ }^{F} R_{i k j l} X^{k} X^{l} .
\end{aligned}
$$

Here the Riemann curvature components are the projections of the Riemann tensor on the tetrad of the observer

$$
{ }^{F} R_{\alpha \beta \gamma \delta}=R_{\mu \nu \rho \sigma} \lambda_{(\alpha)}^{\mu} \lambda_{(\beta)}^{\nu} \lambda_{(\gamma)}^{\rho} \lambda_{(\delta)}^{\sigma}
$$


It turns out that all of the nonzero components of the Riemann tensor in $x^{\mu}=(t, x, y, z)$ coordinates can be obtained from

$$
\begin{aligned}
& R_{0101}=\Omega^{2}, \quad R_{0202}=\Omega^{2} U^{2}(x), \\
& R_{0112}=-\sqrt{2} \Omega^{2} U(x), \quad R_{1212}=3 \Omega^{2} U^{2}(x)
\end{aligned}
$$

using the symmetries of the Riemann tensor. It follows that in the Fermi coordinates all of the nonzero components are given by

$$
{ }^{F} R_{0101}={ }^{F} R_{0202}={ }^{F} R_{1212}=\Omega^{2}
$$

via the symmetries of the Riemann tensor. Thus

$$
\begin{aligned}
& { }^{F} h_{00}=-\Omega^{2}\left(X^{2}+Y^{2}\right), \quad{ }^{F} h_{11}=-\frac{1}{3} \Omega^{2} Y^{2}, \\
& { }^{F} h_{12}={ }^{F} h_{21}=\frac{1}{3} \Omega^{2} X Y, \quad{ }^{F} h_{22}=-\frac{1}{3} \Omega^{2} X^{2},
\end{aligned}
$$

are the only nonzero components of the gravitational potential and the metric at this linear order is given by

$$
\begin{aligned}
d s^{2}= & -\left[1+\Omega^{2}\left(X^{2}+Y^{2}\right)\right] d T^{2}+d X^{2}+d Y^{2}+d Z^{2} \\
& -\frac{1}{3} \Omega^{2}(X d Y-Y d X)^{2} .
\end{aligned}
$$

It is now straightforward to compute the gravitational time delay (4) using the relations $(31)-(32)$. The result is

$$
\begin{aligned}
\Delta_{\text {Gödel }}= & -\frac{1}{6 c^{3}}\left\{\frac{\left[\boldsymbol{\Omega} \cdot\left(\mathbf{R}_{1} \times \mathbf{R}_{2}\right)\right]^{2}}{\left|\mathbf{R}_{1}-\mathbf{R}_{2}\right|}+\left|\mathbf{R}_{1}-\mathbf{R}_{2}\right|\left[\left(\boldsymbol{\Omega} \times \mathbf{R}_{1}\right)^{2}\right.\right. \\
& \left.\left.+\left(\boldsymbol{\Omega} \times \mathbf{R}_{2}\right)^{2}+\left(\boldsymbol{\Omega} \times \mathbf{R}_{1}\right) \cdot\left(\boldsymbol{\Omega} \times \mathbf{R}_{2}\right)\right]\right\}
\end{aligned}
$$

where $\boldsymbol{\Omega}=\Omega \hat{\mathbf{Z}}$ and $\mathbf{R}_{1}$ and $\mathbf{R}_{2}$ are the position vectors of $P_{1}$ and $P_{2}$, respectively.

The relative time delay for a signal from the observer $\left(\mathbf{R}_{1}=0\right)$ to a local distance $R$ compared to $R / c$ is thus given by $(\boldsymbol{\Omega} \times \mathbf{R})^{2} / c^{2}$, which is negligibly small on the basis of the current upper limit of $\Omega \leq 10^{-24} \mathrm{sec}^{-1}$ on the possible rate of rotation of the universe [2], [24]-[27]. 


\section{Gravitomagnetic time delay and gravitational lensing}

In gravitational lensing, depending upon the relative position and distance of the source, observer and deflecting mass, several images of the same source may be observed. Numerous examples of gravitational lensing have been discovered; a well-known case is the gravitational lens Q2237 + 0305, or Einstein Cross, where the path of light from a quasar estimated to be at a distance of approximately 8 billion light years is bent by the gravitational field of a galaxy estimated to be at a distance of about 400 million light years. This light bending produces four images of the same quasar as observed from the Earth [28], [29].

The gravitomagnetic time delay due to the spin of an astrophysical object might then be detected in different images of the same source by gravitational lensing. Time delay in the images may be generated by the spins of the deflecting object and of other bodies around the path of the light rays, e.g. by the spin of an external rotating mass.

Let us estimate the spin time delay corresponding to some astrophysical configurations. For simplicity, to derive the order of magnitude of the gravitomagnetic time delay we assume that the source, lens and observer are aligned. Nevertheless, there is an additional time delay, called geometrical time delay [30], due to the different geometrical paths followed by different rays. Depending on the geometry of the system, this additional term may be very large and may be the main source of time delay. However, if we compare the time delay of photons that follow the same geometrical path we can neglect the geometrical time delay, as in the case of two light rays with the same impact parameter but on different sides of the deflecting object. For a small deflection angle, the contribution to the travel time delay from the deflected path length traveled is of the second order in the deflecting potentials; however, depending on the geometrical configuration considered, this delay may need to be included in the total time delay. Here, for simplicity, we neglect any geometrical time delay. Furthermore, there is a relative time delay due to the quadrupole moment of the central mass distribution. It is shown in [17] how, in the special case of multiple images of the source with the same impact parameter, propagating along the same axis, one may in principle have enough observables to solve for the angular momentum $J$ and mass $M$ of the central deflecting body by eliminating the time delay due to the unknown quadrupole moment. Of course, for other configurations in which the source is not exactly aligned with the lens and the observer, the difference in the paths traveled and the corresponding difference in the Shapiro time delay can be the main source of relative time delay; one would then need to model and remove these delays between the different images on the basis of the observed geometry of the system. 
Let us now calculate the time delay due to the spin of some astrophysical sources. For the Sun, $G M_{\odot} / c^{2} \simeq 1.477 \mathrm{~km}$ and $R_{\odot} \simeq 6.96 \times 10^{5} \mathrm{~km}$, by considering two light rays traveling on the equatorial plane with impact parameters $b \simeq R_{\odot}$ and $-b$, the relative gravitomagnetic time delay from formula (16) is given by $\Delta_{G M}^{\mathrm{rel}}=8 G J /\left(c^{4} b\right) \simeq 1.54 \times 10^{-11}$ sec. The time delay due to spin of the Sun could then, in principle, be measured using an interferometer at a distance of about $8 \times 10^{10} \mathrm{~km}$ by detecting the gravitationally deflected photons emitted by a laser on the side of the Sun opposite the detector and traveling on opposite sides of the Sun to the interferometer. To derive the time delay due to the lensing galaxy of the Einstein Cross [28], [29], we assume a simple model for the rotation and shape of the central object. Details about this model can be found in [31]. The angular separation between the four images is about $0.9^{\prime \prime}$, corresponding to a radius of closest approach of about $650 h_{75}^{-1} \mathrm{pc}$, and the mass inside a shell with this radius is $\sim 1.4 \times 10^{10} h_{75}^{-1} M_{\odot}$ [29]. Let us assume that $G J / c^{3} \simeq 10^{23} h_{75}^{-2} \mathrm{~km}^{2}$, we then have from formula (16) that $\Delta_{G M}^{\text {rel }}=8 G J /\left(c^{4} b\right) \simeq 4 \mathrm{~min}$. Thus, at least in principle, one could measure the time delay due to the spin of the lensing galaxy; of course, as in the case of the Sun, one should be able to model with sufficient accuracy and remove all the other delays due to other physical effects from the observed time delays between the images. As a third example we consider the relative time delay of photons due to the spin of a typical cluster of galaxies of mass $M_{C} \simeq 10^{14} M_{\odot}$, radius $R_{C} \simeq 5 \mathrm{Mpc}$ and angular velocity $\omega_{C} \simeq 10^{-18} \mathrm{sec}^{-1}$; depending on the geometry of the system and on the path followed by the photons, we then find relative time delays ranging from a few minutes to several days $[17]$.

Especially interesting is the case of gravitomagnetic time delay due to the rotation of an external sphere. Let us now calculate the time delay in the travel time of photons propagating inside a rotating shell corresponding to some astrophysical configurations. In the case of the Einstein Cross [28], in order to get an order of magnitude of the effect, we assume that the lensing galaxy has a radius $R \simeq 5 \mathrm{kpc}$; after some calculations based on the model given in [31] and integrating formula (14), the relative time delay of two photons traveling at distances of $650 \mathrm{pc}$ on opposite sides from the center is given by $\Delta_{G M}^{\mathrm{rel}} \simeq 20 \mathrm{~min}$. If the lensing galaxy is inside a rotating cluster, or supercluster of galaxies, to get an order of magnitude of the time delay due to the spin of the mass rotating around the deflecting galaxy, we use typical supercluster parameters: total mass $M=10^{15} M_{\odot}$, radius $R=70 \mathrm{Mpc}$ and angular velocity $\omega=2 \times 10^{-18} \mathrm{sec}^{-1}$ [32]. If the galaxy is in the center of the cluster and light rays have impact parameters $\simeq 15 \mathrm{kpc}$ (of the order of the Milky Way radius), the time delay, in the case of constant density $\rho$ can be determined by integrating formula (14) and the result is $\Delta_{G M}^{\mathrm{rel}} \simeq 1$ day.

Finally, if the lensing galaxy is not at the center of the cluster of radius $R$ but at a distance $a R$ from the center, with $0 \leq a \leq 1$, by integrating formula (14) 
between the impact parameter $r \simeq a R$ and $R$, we find that when the impact parameters $r_{1}$ and $r_{2}$ of two light rays are approximately equal

$$
\Delta_{G M}^{\mathrm{rel}}=\frac{8 G M \omega}{3 c^{4}}\left(r_{1}-r_{2}\right)\left(1-a^{2}\right)^{1 / 2}\left(1-4 a^{2}\right) .
$$

Thus, if the lensing galaxy is at a distance of $10 \mathrm{Mpc}$ from the center of the cluster, the relative time delay due to the spin of the external rotating mass between two photons with $r_{1}-r_{2} \simeq 30 \mathrm{kpc}$ is $\Delta_{G M}^{\mathrm{rel}} \simeq 0.9$ day.

Promising candidates to observe the time delay due to spin are systems of the type of the gravitational lens B0218+357 [33], where the separation angle between the images is so small, 335 milliarcsec for B0218+357, that the time delay between the images is also very small, about 10.5 days for the two images of this system. In such cases, the total time delay is comparable, for a favorable configuration, to the time delay due to spin. In addition, an Einstein ring is observed in the system B0218+357 and the diameter of the ring is the same as the separation of the images. In such a configuration, the Einstein ring can provide strong constraints on the mass distribution in the lens; in turn, this can be extremely useful in order to distinguish time delays of different origin. Since the present measurement uncertainty in the lensing time delay is of the order of 0.5 day, the gravitomagnetic time delay might already be observable [34]. For example in the system B0218+357 the measured delay is $10.5 \pm 0.4$ day. This measurement is possible because the source is a strongly variable radio object, thus one can determine the time delay of the variations in the images. In the case of $\mathrm{B} 0218+357$ it is possible to observe distinct variations in the total flux density, percentage polarization and the polarization position angle at two frequencies.

\section{Discussion}

Time delay plays an important role in classical wave scattering as well as quantum scattering theory (see [35] for a recent comprehensive survey of this subject). Here we have confined our attention to the propagation of radiation in the JWKB limit, i.e. null rays, in a gravitational field that is considered only to linear order. We have then derived expressions for the gravitomagnetic time delay of null rays propagating inside a spinning shell and in the field of rotating masses.

Since we have shown that there may be an appreciable time delay due to the spin of a body, or external shell, we draw the following conclusions.

The gravitomagnetic time delay should be taken into account in the modeling 
of relative time delays of the images of a source observed at a far point by gravitational lensing. This effect is due to the propagation of the photons in opposite directions with respect to the sense of the spin of the body, or external rotating shell.

If other time delays can be modeled with sufficient accuracy and removed from the observational data, the larger relative delay due to the quadrupole moment of the lensing body can be removed, at least in principle and for some configurations of the images, by using special combinations of the observables; thus, one could directly measure the spin time delay due to the gravitomagnetic field of the lensing body. In order to estimate the relevance of the spin time delay in some real astrophysical configurations, we have considered some possible astrophysical cases. We have studied the relative time delay in the gravitational lensing images caused by a typical rotating galaxy or a cluster of galaxies. We have also analyzed the relative gravitomagnetic time delay when the path of photons is inside a galaxy, a cluster or a super-cluster of galaxies rotating around the deflecting body; this effect should be large enough to be detectable from the Earth.

The measurement of the gravitomagnetic time delay due to the angular momentum of an external massive rotating sphere might be a further observable for the determination of the total mass-energy of the external body, i.e. of the dark matter content of galaxies, clusters and super-clusters of galaxies. Indeed, by measuring the gravitomagnetic time delay one can determine the total angular momentum of the rotating body and thus, by estimating the contribution of the visible part, one can determine its dark-matter content.

These estimates are preliminary because we need to apply the gravitomagnetic time delay to some particular, known, gravitational-lensing images. Furthermore, we need to estimate the size and the possibility of modeling of the other sources of time delay. Nevertheless, depending on the geometry of the astrophysical system considered, we conclude that the relative gravitomagnetic time delay may be an already observable effect.

\section{References}

[1] I. I. Shapiro, Phys. Rev. Lett. 13 (1964) 789.

[2] I. Ciufolini and J. A. Wheeler, Gravitation and Inertia (Princeton University Press, Princeton, 1995).

[3] S. A. Klioner, Sov. Astron. 35 (1991) 523.

[4] S. M. Kopeikin, J. Math. Phys. 38 (1997) 2587. 
[5] J. F. Glicenstein, Astron. Astrophys. 343 (1999) 1025.

[6] S. M. Kopeikin, Astrophys. J. Lett. 556 (2001) L1.

[7] S. Kopeikin and B. Mashhoon, Phys. Rev. D 65 (2002) 064025.

[8] B. Linet and P. Teyssandier, Phys. Rev. D 66 (2002) 024045.

[9] B. Mashhoon, Phys. Lett. A 173 (1993) 347.

[10] J. M. Cohen and B. Mashhoon, Phys. Lett. A 181 (1993) 353.

[11] N. Ashby, in: N. Dadhich and J. Narlikar (eds.), Gravitation and Relativity: At the Turn of the Millennium, Proceedings of GR-15 (Inter-University Center for Astronomy and Astrophysics, Pune, India, 1997), pp. 231-258.

[12] N. Ashby, Physics Today 55 (May, 2002) 41.

[13] A. Tartaglia and M. L. Ruggiero, Gen. Rel. Grav. 34 (2002) 1371.

[14] L. Iorio, gr-qc/0201080.

[15] H. Thirring, Z. Phys. 19 (1918) 33.

[16] B. Mashhoon, F. W. Hehl and D. S. Theiss, Gen. Rel. Grav. 16 (1984) 711.

[17] I. Ciufolini and F. Ricci, Class. Quantum Grav. 19 (2002) 3863.

[18] I. Ciufolini and F. Ricci, Class. Quantum Grav. 19 (2002) 3875.

[19] I. Ciufolini and F. Ricci, preprint.

[20] A. Tartaglia and M. L. Ruggiero, preprint.

[21] B. Mashhoon, in: The Big Bang and Georges Lemaitre, edited by A. Berger (Reidel, Dordrecht, 1984), pp. 75-81.

[22] K. Gödel, Rev. Mod. Phys. 21 (1949) 447.

[23] B. Mashhoon, Phys. Rev. D 11 (1975) 2679.

[24] B. Bertotti, I. Ciufolini and P. L. Bender, Phys. Rev. Lett. 58 (1987) 1062.

[25] E. F. Bunn, P. G. Ferreira and J. Silk, Phys. Rev. Lett. 77 (1996) 2883.

[26] Y. N. Obukhov, in: M. Scherfner, T. Chrobok and M. Shefaat (eds.), Colloquium on Cosmic Rotation (Wissenschaft and Technik Verlag, Berlin, 2000), pp. 23-96.

[27] A. Delgado, W. P. Schleich and G. Süssmann, New J. Phys. 4 (2002) 37.

[28] J. Huchra et al., Astron. J. 90 (1985) 691.

[29] R. Racine, Astron. J. 102 (1991) 454.

[30] P. Schneider, J. Ehlers and E. Falco, Gravitational lenses (Springer-Verlag, Berlin, 1992). 
[31] K.-H. Chae, D. A. Turnshek and V. K. Khersonsky, Astrophys. J. 495 (1998) 609.

[32] G. O. Abell, in: Stars and Stellar Systems, IX Galaxies and the Universe (Univ. of Chicago Press, Chicago, 1975), p. 636.

[33] A. D. Biggs, I. W. A. Browne, P. Helbig, L. V. E. Koopmans, P. N. Wilkinson and R. A. Perley, Mon. Not. Roy. Astron. Soc. 304 (1999) 349.

[34] A. D. Biggs and I. W. A. Browne, in: International Astronomical Union Symposium no. 205 (England, August 2000).

[35] C. A. A. de Carvalho and H. M. Nussenzveig, Phys. Rep. 364 (2002) 83. 


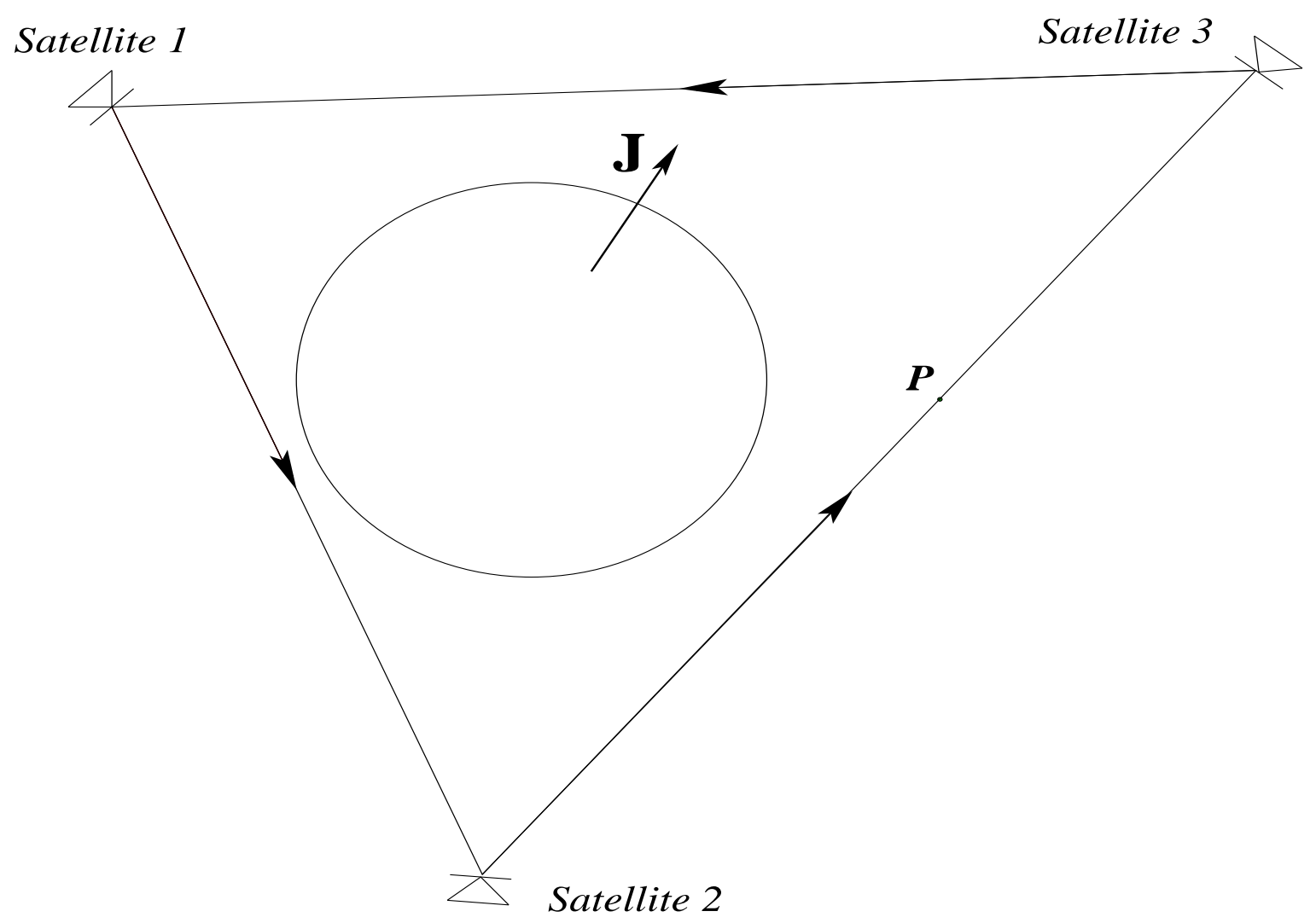

Fig. 1. Light propagates from satellite 1, is transponded from satellites 2 and 3, and returns back to satellite 1 by making a closed counterclockwise loop. Gravitoelectric time delay $\Delta_{G E}$ and gravitomagnetic time delay $\Delta_{G M}$ are due to the gravitational field of the rotating mass $M$ possessing spin $\mathbf{J}$. Point $P$ is an arbitrary point on the loop. The "clock effect" at the point $P$ is given by equation (11) and also discussed in [10] using a different theoretical approach. 